\title{
A comparison of the degree of external root resorption between endodontically treated and its vital contra lateral teeth after orthodontic treatment
}

\begin{abstract}
Introduction: External root resorption (ERR) is a common and often severe multifactorial problem in patients with orthodontic treatment. The excessive and uncontrolled mechanical forces compress the periodontium, which involves resorption cementum caused by the clastic activity.
\end{abstract}

Objective: The purpose of this study was to compare the degree of ERR between endodontically treated teeth and vital contralateral after orthodontic treatment.

Methodology: The sample consisted of evaluating the initial periapical radiographs and the end of 36 patients with an average age between 20-39 years of which had one or more teeth with root canal treatment before starting orthodontic treatment, which was by at least one year. The vital contralateral tooth served as control.

Conclusion: No statistically significant difference in the degree of ERR during orthodontic movement between teeth with root canal therapy and teeth with vital pulp.
Volume 3 Issue I - 2019

\author{
Huízar González Irving Giovanni,' García \\ López Eliezer² \\ 'Graduated University of Guadalajara, Private practice of \\ Orhodontics, Jerez, Zacatecas, México \\ ${ }^{2}$ Professor, Department of Orthodontics, University of \\ Guadalajara, Mexico
}

Correspondence: Irving Giovanni Huízar González, University of Guadalajara, Zacatecas, México, Tel + 5249494235 42, Email Giov_irving@hotmail.com

Received: January 29, 2019 | Published: February II, 2019

Keywords: external root resorption, endodontically treated teeth, orthodontic treatment

\section{Introduction}

External root resorption (ERR) is a physiological or pathological process of lysis that occurs in cement or cement and dentin of the root of a tooth. ${ }^{1}$ Tronstand, states that when there is any mechanical deterioration in dentin or cement, the mineralized or denuded surfaces will be colonized by multinucleated cells and resorption will occur (Figure 1). This process of external root lysis has become one of the crucial problems for dentistry and is usually related to the excessive forces of orthodontic treatment, in addition to involving various factors associated with this pathology. ${ }^{2}$ The ERR is considered an undesirable side effect associated with orthodontic movements, which involves different factors of a biological (related to the patient) and mechanical (related to the treatment) type. Among the biological factors are: genetic factors, chronological age, dental age, nutritional status, gender, race, pharmacological factors, facial and dentoalveolar structure, habits, morphology, size and dental number, dental vitality, previous root resorption, dentoalveolar trauma previous, periapical infections, occlusal factors, specific dental vulnerability to root resorption. Mechanical factors include type of treatment, type of movement, type and magnitude of forces, duration of treatment, severity and type of malocclusion. ${ }^{3}$ The ERR is a consequence of the damage suffered by the periodontal ligament, due to its compression during tooth movement, which comprises a large number of biological effects. The vascular injury of the periodontal ligament gives rise to a phenomenon of aseptic coagulative necrosis known as hyalinization. When a hyalinization occurs, damage to the tissues in the periodontal ligament alters the biochemical environment. The initial reabsorption of the cement begins with the elimination of hyalinized areas by the macrophages that accumulate to eliminate the necrotic tissue. ${ }^{4}$

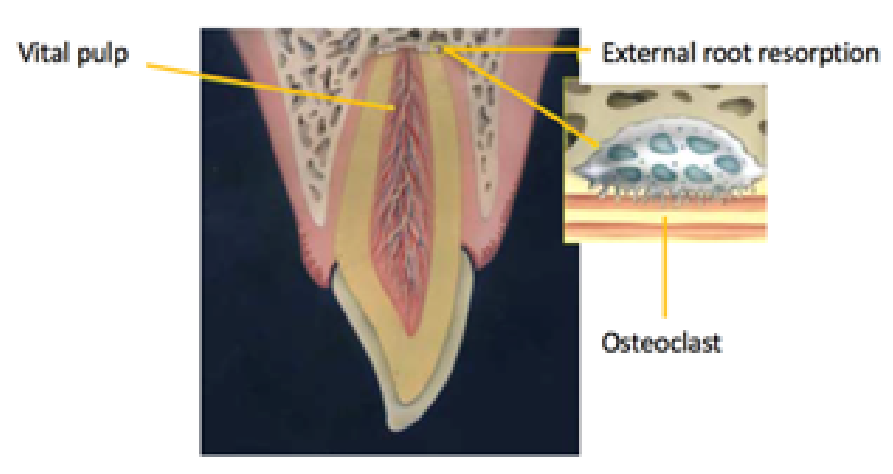

Figure I External root resorption (Modified Root resorption, diagnosis, classification and treatment choices based on stimulation factors, Fuss 2003).

The initial elimination of the hyaline zone occurs at its periphery, where blood flow increases. During the removal of the hyaline zone, the layer of cementoblasts and the cementoide tissue or cement in process of mineralization that covers the root can be damaged and leave exposed the layer of hyalinized cement. It is possible that orthodontic pressure directly damages the layers of the root surface, but still these cells must remove all damaged tissue. The process of resorption continues until the hyaline zone completely disappears or the amount of force decreases due to the decrease in pressure. ${ }^{5}$ ERR is a frequent phenomenon during orthodontic treatment, especially in the upper incisors. In the literature, multiple studies have provided data regarding the number of cases and their frequency. Thus histological studies confirm that in $90 \%$ of cases there is root resorption in teeth subjected to orthodontic treatment. ${ }^{6}$ In 2009 a study was carried out in 
the Department of Orthodontics of the University of Seville, Spain, which consisted in determining the degree of external root resorption in teeth treated with endodontics and teeth with vital pulp as a result of orthodontics. They evaluated 77 patients with fixed appliances for at least one year in treatment, panoramic radiographs were taken before and after. It was found that there was no statistically significant difference between the two groups; the teeth with root canal treatment were reabsorbed $1.00 \mathrm{~mm}$ more than the teeth with vital pulp; however, it was found that there was more resorption in maxillary incisors $1.04+-0.14$ unlike other teeth. ${ }^{7}$ In 2001; Sameshima et al., ${ }^{8}$ They conducted an investigation which consisted in determining which type of radiographs are more accurate to evaluate the shape and root resorption in periapical and panoramic radiographs before and after orthodontic treatment. Radiographs of 42 patients were evaluated before and after having finished their orthodontic treatment

They found that the difference between mandibular incisors in the panoramic radiograph is $1.14 \mathrm{~mm}$, and in the periapical $0.55 \mathrm{~mm}$; The difference between maxillary incisors in the panoramic radiograph is $1.34 \mathrm{~mm}$ and in the periapical $1.21 \mathrm{~mm}$. The average estimated root resorption in all the teeth in the periapical radiograph was 0.48 $\mathrm{mm}$ and in the panoramic radiography it was $0.92 \mathrm{~mm}$, in addition they were able to demonstrate that the panoramic radiography has an elongation of $5-20 \%$, compared to the periapical radiography. The periapical radiograph being the most indicated to evaluate external root resorption. ${ }^{8}$ Parker and Harris in 1998 in Arkansas, evaluated what type of dental movements originated by orthodontics cause greater external root resorption in maxillary central incisors; this study evaluated 110 adolescents with a similar class I malocclusion and bimaxillary protrusion and similar treatment of extractions of four premolars, in addition to using three equal techniques (Tweed, Begg, Roth). No statistically significant difference was found between the techniques; however, they found that there is greater root resorption in root torque, intrusion and translation in retraction of canines and anterior segment.

Studies performed in animal models by Mattison and Mah in 1996, found statistically significant differences of external root resorption between teeth with endodontic treatment $(2.16 \mathrm{~mm}+-1.54)$ and its contralateral with vital pulp $(2.24+-1.58)$, which were submitted to orthodontic forces. ${ }^{10}$ Mirabella and Artun in 1995 in a study of 39 patients who presented teeth with endodontic treatment and their contralaterals with vital pulp, found statistically lower resorption in the teeth with root canal treatments $(0.45 \mathrm{~mm})$, compared to the tooth with vital pulp, in addition of finding a frequency of apical root resorption of $40 \%$ in upper incisors, with more than $2.5 \mathrm{~mm}$ of resorption in adult patients. ${ }^{11}$ Márques, et al. 2010 found a high prevalence, $14.5 \%$, of severe root resorption in patients treated with the edgewise method, compared to the results of other studies. ${ }^{12}$ Brezniak et al, 1993 in his histological studies of extracted teeth with and without root canal treatments, reports that a tooth with pulpal vitality is more reabsorbed $(0.73 \mathrm{~mm})$ compared with a tooth with endodontic or necrotic treatment. This is because the vital tooth contains the nervous nerve complex that gives it the ability to respond actively to the aggressions caused by orthodontic treatment. ${ }^{13}$ According to Linge, et al., ${ }^{14}$ the incidence of ERR is $15 \%$ before orthodontic treatment and $73 \%$ after orthodontic treatment. In terms of severe root resorption, the frequency is relatively low, a $4 \mathrm{~mm}$ loss in root length only occurs in $1-4 \%$ of cases. It is necessary to discern between root resorptions induced by orthodontics, that is, those that are caused by mechanical factors related to orthodontic treatment, and those whose etiology is idiopathic. ${ }^{14}$ In contrast Spurrier et al., ${ }^{15} 1990$ studied 43 patients with root canal treatments. At the end of the treatment it was reported that the incisors with vital pulp were reabsorbed significantly more $(0.77$ $\mathrm{mm}$ ) than the teeth with root canal treatment and greater resorption in men than in women. ${ }^{15}$ The purpose of this study was to compare the degree of ERR between endodontically treated teeth and their contralateral vital after orthodontic treatment.

\section{Subjects and Methods}

The subjects for this investigation were 36 patients ( 22 women and 14 men) with an average age between 20-39 years of whom had one or more teeth with root canal treatment before starting orthodontic treatment which was at least one year. Of which 20 patients were (Class I skeletal), 14 patients (Class II skeletal) and 2 patients (Classes III skeletal).

\section{Inclusion criteria}

Radiographs of patients who have finished orthodontic treatment with fixed appliances of at least one year of treatment and who have at least one tooth with root canal treatment and its contralateral with vital pulp.

\section{Criteria for non-inclusion}

Radiographs of teeth with root fractures, periapical pathologies or teeth that present restoration with crowns.

\section{Radiographic examination and evaluation}

Periapical radiographs were taken for each patient before and after the orthodontic treatment with the use of XCP Dentsply collimators, in order to obtain the most faithful images possible of the dental structures, to avoid the distortion and obtain the best parallelism between the plate and the teeth. The X-ray apparatus of the brand Corix model 70 plus USV-WM was used with an exposure of 0.26 thousandths of a second of the X-rays to the plate. The same radiology assistant and the $\mathrm{x}$-ray machine were used for the two radiographs of each patient. The measurements were taken before and after the orthodontic treatment using Adobe Photoshop CS software. Points and reference lines were placed to perform the measurements (Figure 2). The examination and evaluation of the periapical radiographs was carried out in which the following reference points were used (Figure 3):

\section{Coronal length}

(ICLTET) initial coronal length in tooth with endodontic treatment

(FCLTET) final coronal length in tooth with endodontic treatment

(ICLTVP) initial coronal length in tooth with vital pulp

(FCLTVP) final coronal length in tooth with vital pulp

\section{Radicular length}

(IRLTET) initial root length in tooth with endodontic treatment

(FRLTET) final root length in tooth with endodontic treatment

(IRLTVP) initial root length in tooth with vital pulp

(FRLTVP) final root length in tooth with vital pulp 

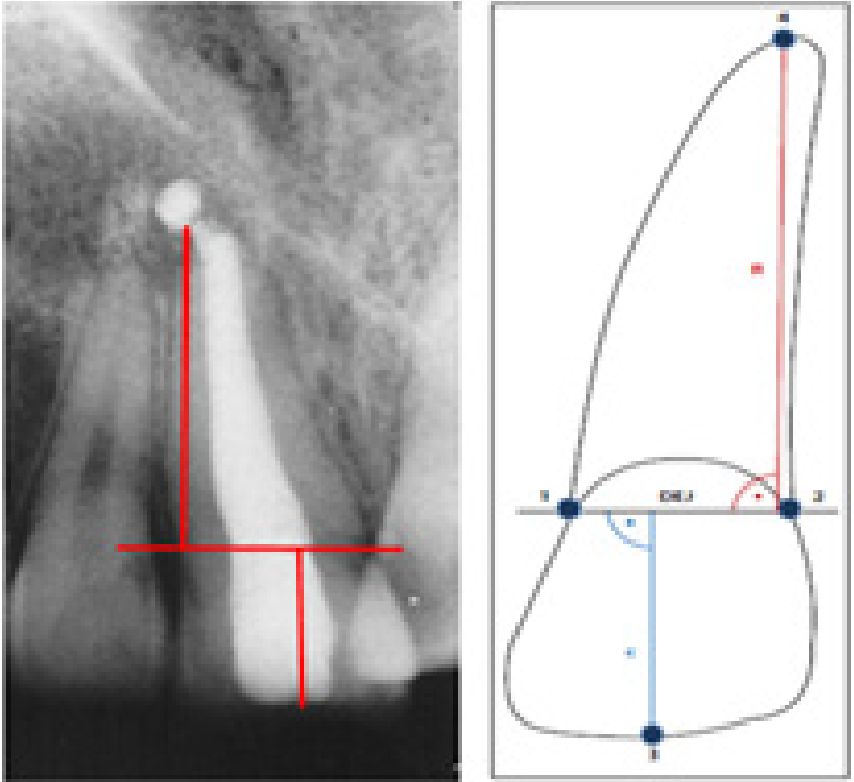

Figure 2 Root crown length with the cement enamel union of reference point (modified Llamas et al., 20I0).

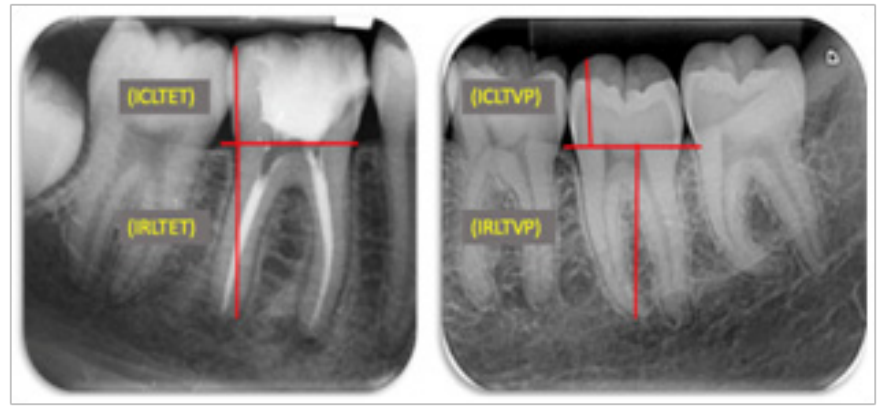

Figure 3 Reference points to evaluate external root resorption (modified Llamas et al., 2010).

To obtain the difference between the external root resorption in the teeth with endodontic treatment and its contralateral with vital pulp before and after the orthodontic treatment a formula is used in which it is important to obtain the product of the division of the length of the crowns before and after the treatment, besides obtaining the difference of the root length; this with the purpose of diminishing the margin of error in the taking of $\mathrm{x}$-rays reflected in an elongated or foreshortened radiograph (Figure 4). ${ }^{7}$

\section{Statistic analysis}

The descriptive statistics of the initial and final root lengths as well as the reduction of the root length due to the orthodontic treatment for both the dental pieces with vital pulp and its contralateral with endodontic treatment were performed. The Student's t-test for independent samples and the Mann-Whitney U-test were applied to compare these parameters between the pieces with vital pulp and its contralateral with endodontic treatment and to analyze more than two groups the ANOVA test to evaluate the decrease of the initial and final root lengths as well as their relationship with age, sex, duration of orthodontic treatment, malocclusion and treatment with and without extractions. All the statistical analysis was carried out considering $\mathrm{p} \leq 0.05$ and as a statistical program we used the software SPSS v. 15.0 (IL, USA) and GraphPad Prism v 5.0 (CA, USA).

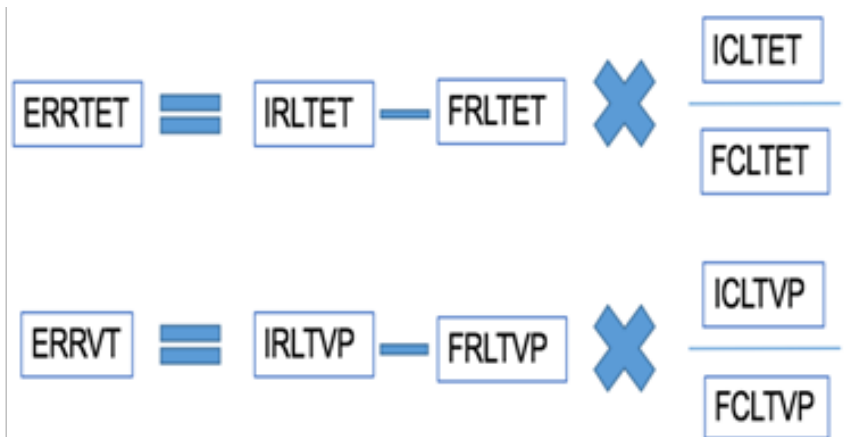

Figure 4 formula for measuring external root resorption in treated endodontics teeth endodontics (ERRTET) and teeth with vital pulp (ERRVT).

\section{Results}

Of the 36 radiographs evaluated, it was found that there is a higher RRE in teeth treated with endodontics $(0.84 \pm 0.48 \mathrm{~mm})$ and lower ERR in teeth with vital pulp $(0.78 \pm 0.42 \mathrm{~mm})$; which is not statistically significant and is considered of little clinical significance (Figure 5). In addition to finding higher ERR in men as opposed to women. On the other hand, according to the observed results, the teeth with the highest ERR incidence were the upper lateral incisors; which several authors have demonstrated as Rupp in $1995 .{ }^{17} \mathrm{~A}$ table of contingencies of age groups by gender and statistical significance is presented with the $x^{2}$ test (Table 1). It is observed that half of the women were located in the age group of 40 years and older; the rest distributed equally among the youngest groups. In men, more than half of them were in the age group of 20 to 39 years, very few in the group of 40 and older and only 4 cases in the younger age group. When evaluating the distribution of cases by age group between sexes with the $x^{2}$ test, no statistically significant differences were observed. In this same table the description of age by sex and total is presented. Women presented an average age of almost 37 years with a large dispersion (standard deviation of 15.1 years), while men had an average age of 27.8 years with less dispersion (standard deviation of 9.0 years). The difference in age between women and men was statistically significant $(\mathrm{p}=0.031)$ with the Student's $\mathrm{t}$ test and also with the Mann-Whitney U test in one tail $(\mathrm{p}=0.043)$.

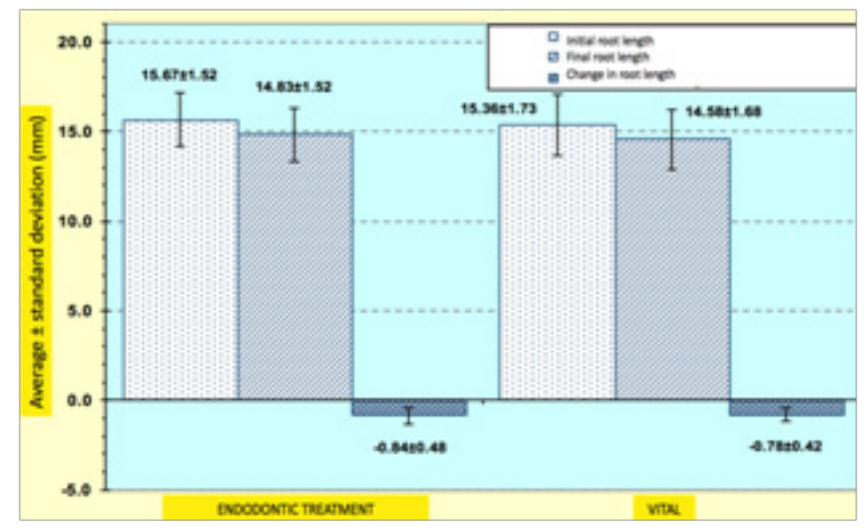

Figure 5 Differences in external root resorption between teeth with endodontic treatment and its contralateral with vital pulp. 
Table I Distribution by age and sex of 36 patients with orthodontic treatment who participated in a study to evaluate the degree of external root resorption in vital teeth and teeth with endodontic treatment. $x^{2}$ test to evaluate differences in the distribution of age between sexes.Average, standard deviation, minimum, maximum and median age by sex and total student $T$ test between sex.

\begin{tabular}{|c|c|c|c|c|c|c|}
\hline \multirow[t]{2}{*}{ Age group } & \multicolumn{3}{|c|}{ Female } & \multicolumn{2}{|l|}{ Male } & \multirow{2}{*}{$\begin{array}{l}\text { Total } \\
\%\end{array}$} \\
\hline & No. & $\%$ & No. & $\%$ & No. & \\
\hline under 20 years & 5 & $22.7 \%$ & 4 & $28.6 \%$ & 9 & $25.0 \%$ \\
\hline 20- 30 years & 6 & $27.3 \%$ & 8 & $57.1 \%$ & 14 & $38.9 \%$ \\
\hline 40 years and older & II & $50.0 \%$ & 2 & $14.3 \%$ & 13 & $36.1 \%$ \\
\hline Total & 22 & $100.0 \%$ & 14 & $100.0 \%$ & 36 & $100.0 \%$ \\
\hline
\end{tabular}

$x^{2}(2)=5.102 ; p=0.078 ; N . S$.

Average \pm standard deviation

(Minimum - maximum)

Median

Student $\mathrm{T}$ test between sexes

$\begin{array}{ll}36.9 \pm 15.1 & 27.8 \pm 9.0 \\ (14-60) & (14-45)\end{array}$

28

$t(33.935)=2.258 ; p=0.031 ; p<0.05$

\section{Discussion}

ERR is an undesirable, and not reversible, pathological process that is related to the outer layers of cement, dentin and root apex. Several meta-analyzes state that teeth with endodontic treatment respond similarly to stimuliy of dental movement during orthodontic treatment. The author Wickwire showed that teeth with endodontic treatment have a higher degree of root resorption. On the other hand, a study by Spurrier has described that some teeth with endodontic treatment are more susceptible to root resorption but not statistically significant than the control group with vital pulp..$^{16}$ The results of this investigation indicate that external root resorption was significantly lower in teeth with vital pulps $(0.78 \pm 0.42 \mathrm{~mm})$ and there is greater external root resorption in teeth with endodontic treatments $(0.84 \pm 0.48$ $\mathrm{mm}$ ); although this difference could be considered of little clinical importance. The result of this study differs from the result of some studies that found a significantly lower root resorption in teeth with root canal treatment undergoing orthodontic treatment, compared to vital teeth. The explanation to the result obtained in this investigation is due to the fact that the ERR is influenced by the osteoclast cells that are in the periodontal ligament, in addition to these teeth that present the endodontic treatment for various reasons such as an infection Periapical trauma or dento - alveolar trauma show greater affinity to the ERR, while orthodontic movement may worsen the tendency to root resorption in previously traumatized teeth. However, in this study an increase in ERR was observed in older patients, although it was not significant, which is attributed to anatomopathological alterations that the periodontal ligament develops over the years. A higher ERR was found in patients with a longer treatment time ( 25 to 36 months), which is due to an application of excessive forces, which suggests that we perform orthodontic treatment in a period of approximately 16 to 24 months. A correlation of higher ERR was found in class I patients who underwent extractions, being lower in class II patients and minimal in class III patients.

\section{Conclusion}

I. ERR is a multifactorial phenomenon, including biological and mechanical factors and this increases its incidence with the poor application of forces in orthodontic treatment.

II. There is a higher ERR in teeth with root canal treatment $(0.84 \pm$
$0.48 \mathrm{~mm})$ and lower in teeth with vital pulp $(0.78 \pm 0.42 \mathrm{~mm})$; which is not statistically significant and is considered of little clinical importance.

III. There is an increased ERR in men, unlike women.

IV. It was shown that the ERR increases when the orthodontic treatment is prolonged after 25 months of treatment.

V. ERR prevails in class I patients with extractions treatment.

VI. The absence of vital pulp in teeth treated with endodontics is not a predisposing factor of the ERR, however, it is the stimulation of the forces to the periodontal ligament.

\section{Acknowledgments}

None.

\section{Conflicts of interest}

The author declares there is no conflict of interest.

\section{References}

1. Levander E, Malmgren O, Stenback K. Apical root resorption during orthodontic treatment of patients with multiple aplasia: a study of maxillary incisors. Eur J Orthod. 1998;20(4):427-434.

2. Tronstad L. Root resorption-etiology, terminology and clinical manifestations. Endod Dent Traumatol. 1998;4(6):241-251.

3. Uribe Restrepo GA. Ortodoncia: teoría y clínica. $2^{\text {nd }}$ edition. Medellín: CIB; 2010.

4. Mavragani M, Brudvik P, Selvig KA. Orthodontically induced root and alveolar bone resorption: inhibitory effect of systemic doxycycline administration in rats. Eur J Orthod. 2005;27(3):215-225.

5. Jäger A, Kunert D, Friesen T, et al. Cellular and extracellular factors in early root resorption repair in the rat. Eur J Orthod. 2008;30(4):336-345.

6. Vaquero, P. Reabsorción radicular durante el tratamiento ortodóncico: Causas y recomendaciones de actuación. Cient Dent. 2011;8:61-70.

7. Llamas-Carreras J, Amarilla A, Solano E, et al. Study of external root resorption during orthodontic treatment in root filled teeth compared with their contralateral teeth with vital pulps. Int Endod J. 2010;43(8):654 662 . 
8. Sameshima, G. Assessment of Root Resorption and Root Shape: Periapical vs Panoramic Films. Angle Orthod. 2001;71(3):185-189.

9. Parker R, Harris E. Directions of orthodontic tooth movements associated with external apical root resorption of the maxillary central incisor. $\mathrm{Am} \mathrm{J}$ Orthod Dentofacial Orthop. 1998;114(6):677-683.

10. Mattison GD, Delivanis HP, Delivanis PD, et al. Orthodontic root resorption of vital and endodontically treated teeth. Journal of Endodontics. 1984;10:354-358.

11. Mirabella AD, Artun J. Prevalence and severity of apical root resorption of maxillary anterior teeth in adult orthodontic patients. Eur J Orthod. 1995;17(2):93-99.

12. Marques LS, Ramos-Jorge ML, Rey AC, et al. Severe root resorption in orthodontic patients treated with the edgewise method: Prevalence and predictive factors. Am J Orthod Dentofacial Orthop. 2010;137(3):384388
13. Brezniak N, Wasserstein A. Orthodontically induced inflammatory root resorption: Part II. The clinical aspects. Angle Orthod. 2002;72(2):180 184.

14. Linge L. Patient characteristics and treatment variables associated with apical root resorption during orthodontic treatament. Am J Orthod Dentofacial Orthop. 1991;99(1):35-43.

15. Spurrier, Steven. A comparison of apical root resorption during orthodontic treatment in endodontically treated and vital teeth. Am J Orthod Dentof Orthop. 1990;97(2):130-134.

16. Wickwire NA, McNeil MH, Norton LA, et al. The effects of tooth movement upon endodontically treated teeth. Angle Orthbd. 1974;44:235-242.

17. Rupp R. Root resortption related to orthodontic and other factors: A review of the literature. J Gen Orthod. 1995;6(3):25-29. 